\title{
Pazopanib in Soft Tissue Sarcomas
}

\author{
An Expert Interview with Brian Van Tine
}

Washington University, St Louis, MO, USA

DOI: https://doi.org/10.17925/OHR.2020.16.1.15

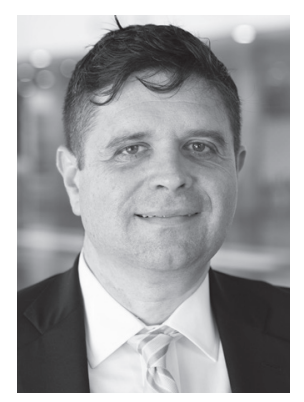

Brian Van Tine

Brian Van Tine is Assistant Professor of Medicine in the Department of Medicine at Washington University in St Louis, MO, USA. He is the Sarcoma Program Director at the Alvin I Siteman Cancer Center. He received his BSC from the Departments of Chemistry and Biochemistry at the University of Arizona in 1995. He completed his MD and PhD at the University of Alabama at Birmingham in 2005. He then came to Washington University in St Louis/Barnes-Jewish Hospital, where he completed an Internal Medicine Residency and Medical Oncology Fellowship. He is the sarcoma medical oncologist for the mid-west region surrounding St Louis, focusing his efforts on translating basic science observations to clinical trials. His laboratory is dedicated to understanding the metabolomic deficiencies found in sarcomas, as they can be therapeutically targeted. His laboratory has identified argininosuccinate synthetase 1 deficiencies in sarcomas that can be treated with pegylated-arginine deiminase. Among other research is the development of biomarker-driven metabolic therapies for all sarcomas, and identification of a metabolic deficiency in synovial sarcoma that can also be therapeutically exploited.

\section{Keywords}

Pazopanib, soft tissue sarcoma

Disclosures: Brian Van Tine has received basic science grant funding from Pfizer, Tracon, and Merck; consulting fees from Epizyme, Eli Lilly, CytRX, Janssen, Immune Design, Daiichi Sankyo, Plexxicon, and Adaptimmune; speaking fees from Caris, Janseen, and Eli Lilly, and is the overall principal investigator on NCT03449901.

Acknowledgment: Medical writing assistance was provided by Katrina Mountfort of Touch Medical Media, and supported by Touch Medical Media.

Review Process: This is an expert interview and as such, has not undergone the journal's standard peer review process.

Compliance with Ethics: This is an expert interview and does not report on new clinical data, or any studies with human or animal subjects performed by the author.

Authorship: The named author meets the International Committee of Medical Journal Editors (ICMJE) criteria for authorship of this manuscript, takes responsibility for the integrity of the work as a whole, and has given final approval to the version to be published.

Access: This article is freely accessible at touchONCOLOGY.com (c) Touch Medical Media 2020.

Received: October 16, 2019

Published Online: April 22, 2020

Citation: Oncology \& Hematology Review (US). 2020;16(1):15-6

Corresponding Author: Brian Van Tine,

Medical Oncology, Room 3302, 3rd Floor McKinley

Building, Barnes and Jewish Hospital, Washington

University in St Louis, Box 8076, 660 South Euclid Avenue,

St Louis, MO 63110, USA.

E: bvantine@wustl.edu

Support: No funding was received in the publication of this article.
S oft tissue sarcomas represent a group of over 80 rare malignant tumors that arise from tissues of mesenchymal origin throughout the body. ${ }^{1}$ Advanced soft tissue sarcoma is treated with single-agent or combination systemic chemotherapy, but is associated with a poor prognosis. ${ }^{2,3}$ Pazopanib (Votrient ${ }^{\circledR}$, Novartis, Basel, Switzerland) is an oral multitarget tyrosine kinase inhibitor that has received regulatory approval as a second-line and beyond treatment for metastatic soft tissue sarcoma based on the findings of the phase III PALETTE study (ClinicalTrials.gov identifier: NCT00753688). ${ }^{4}$ However, there is a population of elderly and debilitated patients with soft tissue sarcoma who are not fit for standard first-line chemotherapy that is anthracycline-based. As pazopanib is well tolerated with minimal side effects, a phase II study (ClinicalTrials.gov identifier: NCT02300545) investigated the use of pazopanib as front-line therapy in patients with non-resectable or metastatic soft tissue sarcomas who are not candidates for chemotherapy. ${ }^{5}$

At the European Society for Medical Oncology (ESMO) meeting in 2019, which was held from September 27-October 1 in Barcelona, Spain, Dr. Van Tine discussed the use of pazopanib and the findings of the study.

\section{Q. What are the limitations of standard first-line chemotherapy in the treatment of soft tissue sarcomas?}

The front-line therapy of soft-tissue sarcomas has not changed since the 1970s. ${ }^{3}$ Following promising findings in a phase I study, ${ }^{6}$ we hoped that olaratumab might be an active and useful drug, but the phase III ANNOUNCE trial showed no benefit.' Front-line chemotherapy is toxic in many patients; therefore, this is an important unmet need.

\section{Q. What is the rationale for the use of pazopanib as first-line therapy in patients with non-resectable or metastatic soft tissue sarcomas?}

When pazopanib first came on the market, one of the ways in which it was used was lowering the dose and then escalating. We found that this was a more tolerable medication compared with doxorubicin, so we investigated it in a patient population that may not tolerate chemotherapy, such as debilitated and elderly patients with soft tissue sarcoma. 


\section{Q. Could you tell us a little about the phase II study of pazopanib and its efficacy and safety findings?}

The study involved 56 patients who were at least 18 years old, not eligible for chemotherapy, and had not received prior systemic therapy for soft tissue sarcoma. Pazopanib was initiated in patients at a dose of $200 \mathrm{mg}$ twice daily for 4 days, escalated to a dose of $400 \mathrm{mg}$ twice daily for 4 days, then escalated once more to a dose of $800 \mathrm{mg}$ twice daily for the duration of the trial. Results showed that a subset of patients showed benefits in terms of progression-free survival and overall survival. There were no significant changes in quality of life whether patients were responders or not. ${ }^{5}$ No new or unexpected adverse events were seen. A European phase ॥ study comparing pazopanib and doxorubicin showed similar findings. ${ }^{8}$

\section{Q. Which patients are most likely to benefit from this treatment approach?}

Patients who want to try something but are afraid to try chemotherapy are most likely to benefit from this approach. Certain subtypes of sarcoma such as synovial sarcoma also respond.

\section{Q. How common is pazopanib resistance and how might this be overcome?}

Most cases of sarcoma eventually become resistant to pazopanib. ${ }^{9}$ The data from our study shows that pazopanib is successful in two out of five patients. The incidence of pazopanib resistance is the same whether it is used as front-line or later-line therapy. ${ }^{5} \square$

1. Casali PG, Abecassis N, Aro HT, et al. Soft tissue and visceral sarcomas: ESMO-EURACAN Clinical Practice Guidelines for diagnosis, treatment and follow-up. Ann Oncol. 2018;29:(Suppl. 4):iv51-67.

2. Van Glabbeke M, van Oosterom AT, Oosterhuis JW, et al. Prognostic factors for the outcome of chemotherapy in advanced soft tissue sarcoma: an analysis of 2,185 patients treated with anthracycline-containing first-line regimens-a European Organization for Research and Treatment of Cancer Soft Tissue and Bone Sarcoma Group Study. J Clin Oncol. 1999;17:150-7.

3. Benjamin RS, Wiernik PH, Bachur NR. Adriamycin: a new effective agent in the therapy of disseminated sarcomas. Med Pediatr Oncol. 1975;1:63-76.

4. van der Graaf WT, Blay JY, Chawla SP, et al. Pazopanib for metastatic soft-tissue sarcoma (PALETTE): a randomised, double-blind, placebo-controlled phase 3 trial. Lancet. 2012;379:1879-86.

5. Hirbe A, Luo J, Seetharam M, et al. 1677PD A phase II study of pazopanib as front-line therapy in patients with non-resectable or metastatic soft tissue sarcomas who are not candidates for chemotherapy. Ann Oncol. 2019;30(Suppl. 5):v688.

6. Tap WD, Jones RL, Van Tine BA, et al. Olaratumab and doxorubicin versus doxorubicin alone for treatment of soft-tissue sarcoma: an open-label phase 1b and randomised phase 2 trial. Lancet. 2016;388:488-97.

7. The ASCO Post. Results reported from the phase III ANNOUNCE trial of olaratumab in soft-tissue sarcoma. 2019. Available at: www.ascopost.com/News/59688 (accessed March 11, 2020).

8. Karch A, Koch A, Grünwald V. A phase II trial comparing pazopanib with doxorubicin as first-line treatment in elderly patients with metastatic or advanced soft tissue sarcoma (EPAZ): study protocol for a randomized controlled trial. Trials. 2016;17:312.

9. Lee ATJ, Jones RL, Huang PH. Pazopanib in advanced soft tissue sarcomas. Signal Transduct Target Ther. 2019;4:16. 